\title{
Symbolic Models for Randomly Switched Stochastic Systems
}

\author{
Majid Zamani and Alessandro Abate
}

\begin{abstract}
In the past few years, there has been a growing interest in the use of symbolic models for control systems, however this has only recently covered the class of continuoustime stochastic hybrid systems. The main reason for this interest is the possibility to use algorithmic techniques over symbolic models to synthesize hybrid controllers enforcing logic specifications on the original models, which would be hard (or even impossible) to enforce with classical control techniques. Examples of such specifications include those expressible via linear temporal logic or as automata on infinite strings. The main challenge in this research line is in the identification of classes of systems that admit symbolic models. In this work we progress in this direction by enlarging the class of stochastic hybrid systems admitting such models: in particular we show that randomly switched stochastic systems, satisfying some incremental stability assumption, admit symbolic models.
\end{abstract}

\section{INTRODUCTION}

Stochastic hybrid systems represent a general class of dynamical systems that combine continuous dynamics with discrete components and that are affected by probabilistic noise and discrete events. There are many real-life systems from fields such as air traffic control [6], systems biology [9], and communication networks [8], that can be modeled as stochastic hybrid systems. Randomly switched stochastic systems, also known as switching stochastic systems, are a relevant sub-class of general stochastic hybrid systems. They consist of a finite family of subsystems (modes or locations), together with an adversarial random switching signal that specifies the active subsystem at every instant of time. Each subsystem is further endowed with continuous probabilistic dynamics that are described by a control-dependent stochastic differential equation.

Recently, there has been some research focused on characterizing classes of systems, involving continuous (possibly hybrid) components, that admit symbolic models. A symbolic model is a discrete approximation of a concrete model resulting from replacing equivalent continuous states by finite symbols. Symbolic models are interesting because they allow the application of the algorithmic machinery for controller synthesis over discrete systems [16] towards the synthesis of controllers for the original models. Such controllers are synthesized to satisfy classes of specifications that traditionally have not been considered in the context of control theory: these include specifications involving regular languages and temporal logic.

This work is supported by the European Commission STREP project MoVeS 257005 and IAPP project AMBI 324432.

M. Zamani is with the Department of Design Engineering, Delft University of Technology, 2628 CE, Delft, The Netherlands. A. Abate is with the Department of Computer Science, University of Oxford, Oxford, OX1 3QD, United Kingdom. Email: m.zamani@tudelft.nl, alessandro.abate@cs.ox.ac.uk.
The search for classes of continuous-time stochastic (hybrid) systems admitting symbolic models include results on stochastic dynamical systems under contractivity assumptions [1], which are valid only for autonomous models (i.e. with no control input); on probabilistic rectangular automata [20] with random behaviors exclusively over the discrete components and with simple continuous dynamics; on linear stochastic control systems [14], however without providing any quantitative relationship between abstract and concrete model; on stochastic control systems without any stability assumption and no hybrid dynamics [24]; on incrementallystable stochastic control systems without discrete components [25] and without requiring state-space discretization [26], and finally on incrementally-stable stochastic switched systems [23] in which the discrete dynamics, in the form of mode changes, are governed by a non-probabilistic control signal. The results in [23], [24], [25], [26] are based on the notion of (alternating) approximate (bi)simulation relation, as discussed in [5], [19]. Notions of bisimulation for continuous-time stochastic hybrid systems have also been studied in [10], although with a purpose different than the synthesis of finite abstractions. Indeed, while we are interested in the construction of bisimilar models that are finite, the work in [10] uses bisimulation to relate continuous (and thus infinite) stochastic hybrid systems. Finally, there exist discretization results based on weak approximations of continuous-time stochastic control systems [13] and of continuous-time stochastic hybrid systems [12], however these do not provide any explicit approximation bound.

To the best of our knowledge there is no work on the construction of finite bisimilar abstractions for continuoustime switching stochastic systems for which the discrete dynamics, in the form of mode changes, are governed by an adversarial random switching signal. Models for these systems have become almost ubiquitous in engineering applications such as power electronics [21], economic and finance [7]: automated controller synthesis techniques can result in more reliable system development at lower costs and times.

The main contribution of this paper is to show that switching stochastic systems, under some incremental stability assumption, admit symbolic models that are alternatingly approximately bisimilar to the concrete models, with a precision $\varepsilon$ that can be chosen a-priori, as a design parameter. By guaranteeing the existence of an alternating $\varepsilon$-approximate bisimulation relation between concrete and symbolic models, one deduces that there exists a controller enforcing a desired specification on the symbolic model if and only if there exists a controller enforcing an $\varepsilon$-related specification on the original switching stochastic system. Due to space constraints, most of the proofs of the main results 
are omitted from this manuscript and will appear elsewhere.

\section{RAndomly Switched Stochastic Systems}

\section{A. Notation}

The identity map on a set $A$ is denoted by $1_{A}$. If $A$ is a subset of $B$, we denote by $\imath_{A}: A \hookrightarrow B$ or simply by $\imath$ the natural inclusion map taking any $a \in A$ to $\imath(a)=a \in B$. The symbols $\mathbb{N}, \mathbb{N}_{0}, \mathbb{Z}, \mathbb{R}, \mathbb{R}^{+}$, and $\mathbb{R}_{0}^{+}$denote the set of natural, nonnegative integer, integer, real, positive, and nonnegative real numbers, respectively. The symbols $0_{n}$ and $0_{n \times m}$ denote the zero vector and matrix in $\mathbb{R}^{n}$ and $\mathbb{R}^{n \times m}$, respectively. Given a vector $x \in \mathbb{R}^{n}$, we denote by $x_{i}$ the $i-$ th element of $x$, and by $\|x\|$ the infinity norm of $x$, namely, $\|x\|=\max \left\{\left|x_{1}\right|,\left|x_{2}\right|, \ldots,\left|x_{n}\right|\right\}$, where $\left|x_{i}\right|$ denotes the absolute value of $x_{i}$. Given matrices $M=\left\{m_{i j}\right\} \in \mathbb{R}^{n \times m}$ and $P=\left\{p_{i j}\right\} \in \mathbb{R}^{n \times n}$, we denote by $\|M\|$ the infinity norm of $M$, namely, $\|M\|=\max _{1 \leq i \leq n} \sum_{j=1}^{m}\left|m_{i j}\right|$, and by $\operatorname{Tr}(P)$ the trace of $P$, namely, $\operatorname{Tr}(P)=\sum_{i=1}^{n} p_{i i}$. We denote by $\Delta$ the diagonal set, namely, $\Delta=\left\{(x, x) \mid x \in \mathbb{R}^{n}\right\}$.

The closed ball centered at $x \in \mathbb{R}^{n}$ with radius $\lambda$ is defined by $\mathcal{B}_{\lambda}(x)=\left\{y \in \mathbb{R}^{n} \mid\|x-y\| \leq \lambda\right\}$. A set $B \subseteq \mathbb{R}^{n}$ is called a box if $B=\prod_{i=1}^{n}\left[c_{i}, d_{i}\right]$, where $c_{i}, d_{i} \in \mathbb{R}$ with $c_{i}<$ $d_{i}$ for each $i \in\{1, \ldots, n\}$. The span of a box $B$ is defined as $\operatorname{span}(B)=\min \left\{\left|d_{i}-c_{i}\right| \mid i=1, \ldots, n\right\}$. By defining $\left[\mathbb{R}^{n}\right]_{\eta}=\left\{a \in \mathbb{R}^{n} \mid a_{i}=k_{i} \eta, k_{i} \in \mathbb{Z}, i=1, \ldots, n\right\}$, the set $\bigcup_{p \in\left[\mathbb{R}^{n}\right]_{\eta}} \mathcal{B}_{\lambda}(p)$ is a countable covering of $\mathbb{R}^{n}$ for any $\eta \in$ $\mathbb{R}^{+}$and $\lambda \geq \eta / 2$. For a box $B$ and $\eta \leq \operatorname{span}(B)$, define the $\eta$-approximation $[B]_{\eta}=\left[\mathbb{R}^{n}\right]_{\eta} \cap B$. Note that $[B]_{\eta} \neq \varnothing$ for any $\eta \leq \operatorname{span}(B)$. Geometrically, for any $\eta \in \mathbb{R}^{+}$with $\eta \leq$ $\operatorname{span}(B)$ and $\lambda \geq \eta$, the collection of sets $\left\{\mathcal{B}_{\lambda}(p)\right\}_{p \in[B]_{\eta}}$ is a finite covering of $B$, i.e., $B \subseteq \bigcup_{p \in[B]_{\eta}} \mathcal{B}_{\lambda}(p)$. We extend the notions of span and approximation to finite unions of boxes as follows. Let $A=\bigcup_{j=1}^{M} A_{j}$, where each $A_{j}$ is a box. Define $\operatorname{span}(A)=\min \left\{\operatorname{span}\left(A_{j}\right) \mid j=1, \ldots, M\right\}$, and for any $\eta \leq \operatorname{span}(A)$, define $[A]_{\eta}=\bigcup_{j=1}^{M}\left[A_{j}\right]_{\eta}$.

We denote by $\mathbf{d}_{h}$ the Hausdorff pseudometric induced by a metric $\mathbf{d}$ on $2^{X}$; we recall that for any $X_{1}, X_{2} \subseteq$ $X, \mathbf{d}_{h}\left(X_{1}, X_{2}\right):=\max \left\{\overrightarrow{\mathbf{d}}_{h}\left(X_{1}, X_{2}\right), \overrightarrow{\mathbf{d}}_{h}\left(X_{2}, X_{1}\right)\right\}$, where $\overrightarrow{\mathbf{d}}_{h}\left(X_{1}, X_{2}\right)=\sup _{x_{1} \in X_{1}} \inf _{x_{2} \in X_{2}} \mathbf{d}\left(x_{1}, x_{2}\right)$ is the directed Hausdorff pseudometric. Given a measurable function $f: \mathbb{R}_{0}^{+} \rightarrow \mathbb{R}^{n}$, the (essential) supremum (sup norm) of $f$ is denoted by $\|f\|_{\infty}$; we recall that $\|f\|_{\infty}=($ ess $) \sup \{\|f(t)\|, t \geq 0\}$. A continuous function $\gamma: \mathbb{R}_{0}^{+} \rightarrow \mathbb{R}_{0}^{+}$, is said to belong to class $\mathcal{K}$ if it is strictly increasing and $\gamma(0)=0 ; \gamma$ is said to belong to class $\mathcal{K}_{\infty}$ if $\gamma \in \mathcal{K}$ and $\gamma(r) \rightarrow \infty$ as $r \rightarrow \infty$. A continuous function $\beta: \mathbb{R}_{0}^{+} \times \mathbb{R}_{0}^{+} \rightarrow \mathbb{R}_{0}^{+}$is said to belong to class $\mathcal{K} \mathcal{L}$ if, for each fixed $s$, the map $\beta(r, s)$ belongs to class $\mathcal{K}$ with respect to $r$ and, for each fixed nonzero $r$, the map $\beta(r, s)$ is decreasing with respect to $s$ and $\beta(r, s) \rightarrow 0$ as $s \rightarrow \infty$. We identify a relation $R \subseteq A \times B$ with the map $R: A \rightarrow 2^{B}$ defined by $b \in R(a)$ iff $(a, b) \in R$. Given a relation $R \subseteq A \times B, R^{-1}$ denotes the inverse relation defined by $R^{-1}=\{(b, a) \in B \times A:(a, b) \in R\}$.

\section{B. Randomly switched (a.k.a. switching) stochastic systems}

Let $(\Omega, \mathcal{F}, \mathbb{P})$ be a probability space endowed with a filtration $\mathbb{F}=\left(\mathcal{F}_{t}\right)_{t \geq 0}$ satisfying the usual conditions of completeness and right-continuity $[11, \mathrm{p} .48]$. Let $\left\{W_{t}\right\}_{t \geq 0}$ be a $\widehat{q}$-dimensional F-Brownian motion [18].

Definition 2.1: A switching stochastic system is a tuple $\Sigma=\left(\mathbb{R}^{n}, \mathrm{U}, \mathcal{U}, \mathrm{P}, \mathcal{P}, F, G\right)$, where

- $\mathbb{R}^{n}$ is the state space;

- $U \subseteq \mathbb{R}^{m}$ is a compact input set;

- $\mathcal{U}$ is a subset of the set of all measurable functions of time from $\mathbb{R}_{0}^{+}$to $\mathrm{U}$

- $\mathrm{P}=\{1, \ldots, m\}$ is a finite set of modes;

- $\mathcal{P}$ is a subset of the set of all piecewise constant càdlàg (i.e. right-continuous and with left limits) functions of time from $\mathbb{R}_{0}^{+}$to $P$, and characterized by a finite number of discontinuities on every bounded interval in $\mathbb{R}_{0}^{+}$(this ensures no Zeno behavior);

- $F=\left\{f_{1}, \ldots, f_{m}\right\}$ is such that, for all $p \in \mathrm{P}, f_{p}$ : $\mathbb{R}^{n} \times \mathrm{U} \rightarrow \mathbb{R}^{n}$ is globally Lipschitz continuous;

- $G=\left\{g_{1}, \ldots, g_{m}\right\}$ is such that, for all $p \in \mathrm{P}, g_{p}: \mathbb{R}^{n} \rightarrow$ $\mathbb{R}^{n \times \widehat{q}}$ is globally Lipschitz continuous with a Lipschitz constant $Z \in \mathbb{R}^{+}$.

A continuous-time stochastic process $\xi: \Omega \times \mathbb{R}_{0}^{+} \rightarrow \mathbb{R}^{n}$ is said to be a solution process of $\Sigma$ if there exist $\pi \in \mathcal{P}$ and $v \in \mathcal{U}$ satisfying

$$
\mathrm{d} \xi=f_{\pi}(\xi, v) \mathrm{d} t+g_{\pi}(\xi) \mathrm{d} W_{t},
$$

$\mathbb{P}$-almost surely (P-a.s.) at each time $t \in \mathbb{R}_{0}^{+}$where $\pi$ is continuous. For any given $p \in \mathrm{P}$, we denote by $\Sigma_{p}$ the subsystem of $\Sigma$ defined by the stochastic differential equation

$$
\mathrm{d} \xi=f_{p}(\xi, v) \mathrm{d} t+g_{p}(\xi) \mathrm{d} W_{t},
$$

for any $v \in \mathcal{U}$, where $f_{p}$ is known as the drift and $g_{p}$ as the diffusion. A solution process of $\Sigma_{p}$ exists and is uniquely determined owing to the assumptions on $f_{p}$ and on $g_{p}[18$, Theorem 5.2.1, p. 68].

In this paper, we assume that $\pi$ randomly dictates in which mode the solution process $\xi$ is at any time $t \in \mathbb{R}_{0}^{+}$.

We further write $\xi_{a v}^{\pi}(t)$ to denote the value of the solution process at time $t \in \mathbb{R}_{0}^{+}$under the control input $v \in \mathcal{U}$ and the switching signal $\pi$ from initial condition $\xi_{a v}^{\pi}(0)=a$ $\mathrm{P}$-a.s., in which $a$ is a random variable that is measurable in $\mathcal{F}_{0}$. Note that a solution process of $\Sigma_{p}$ is also a solution process of $\Sigma$ corresponding to the constant switching signal $\pi(t)=p$, for all $t \in \mathbb{R}_{0}^{+}$. We also use $\xi_{a v}^{p}(t)$ to denote the value of the solution process of $\Sigma_{p}$ at time $t \in \mathbb{R}_{0}^{+}$under the control input $v \in \mathcal{U}$ from the initial condition $\xi_{a v}^{p}(0)=a$ P-a.s..

\section{A Notion of InCREMEnTAL StABility}

The main result presented in this paper requires a certain stability property on $\Sigma$, which is inspired by the one introduced in [2] and formally defined next.

Definition 3.1: A switching stochastic system $\Sigma$ is incrementally globally asymptotically stable in the $q$ th moment $\left(\delta\right.$-GAS-M $\left.{ }_{q}\right)$, where $q \geq 1$, if there exists a $\mathcal{K} \mathcal{L}$ function $\beta$ such that for any $t \in \mathbb{R}_{0}^{+}$, any $\mathbb{R}^{n}$-valued random variables 
$a$ and $a^{\prime}$ that are measurable in $\mathcal{F}_{0}$, any $v \in \mathcal{U}$, and any $\pi \in \mathcal{P}$, the following condition is satisfied:

$$
\mathbb{E}\left[\left\|\xi_{a v}^{\pi}(t)-\xi_{a^{\prime} v}^{\pi}(t)\right\|^{q}\right] \leq \beta\left(\mathbb{E}\left[\left\|a-a^{\prime}\right\|^{q}\right], t\right) .
$$

One can describe $\delta$-GAS-M $\mathrm{M}_{q}$ in terms of the existence of so-called incremental Lyapunov functions, as defined next.

Definition 3.2: Consider a stochastic subsystem $\Sigma_{p}$ and a continuous function $V_{p}: \mathbb{R}^{n} \times \mathbb{R}^{n} \rightarrow \mathbb{R}_{0}^{+}$that is twice continuously differentiable on $\left\{\mathbb{R}^{n} \times \mathbb{R}^{n}\right\} \backslash \Delta$. Function $V_{p}$ is called a $\delta$-GAS-M $\mathrm{M}_{q}$ Lyapunov function for $\Sigma_{p}$, where $q \geq 1$, if there exist $\mathcal{K}_{\infty}$ functions $\underline{\alpha}_{p}, \bar{\alpha}_{p}$, and a constant $\kappa_{p} \in \mathbb{R}^{+}$, such that

(i) $\underline{\alpha}_{p}$ (resp. $\bar{\alpha}_{p}$ ) is a convex (resp. concave) function;

(ii) for any $x, x^{\prime} \in \mathbb{R}^{n}$,

$$
\underline{\alpha}_{p}\left(\left\|x-x^{\prime}\right\|^{q}\right) \leq V_{p}\left(x, x^{\prime}\right) \leq \bar{\alpha}_{p}\left(\left\|x-x^{\prime}\right\|^{q}\right)
$$

(iii) for any $x, x^{\prime} \in \mathbb{R}^{n}$, such that $x \neq x^{\prime}$, and any $u \in \mathrm{U}$,

$$
\begin{aligned}
& \mathcal{L}^{u} V_{p}\left(x, x^{\prime}\right):=\left[\begin{array}{ll}
\partial_{x} V_{p} & \partial_{x^{\prime}} V_{p}
\end{array}\right]\left[\begin{array}{c}
f_{p}(x, u) \\
f_{p}\left(x^{\prime}, u\right)
\end{array}\right]+ \\
& \frac{1}{2} \operatorname{Tr}\left(\left[\begin{array}{c}
g_{p}(x) \\
g_{p}\left(x^{\prime}\right)
\end{array}\right]\left[g_{p}^{T}(x) g_{p}^{T}\left(x^{\prime}\right)\right]\left[\begin{array}{cc}
\partial_{x, x} V_{p} & \partial_{x, x^{\prime}} V_{p} \\
\partial_{x^{\prime}, x} V_{p} & \partial_{x^{\prime}, x^{\prime}} V_{p}
\end{array}\right]\right) \\
& \leq-\kappa_{p} V_{p}\left(x, x^{\prime}\right) \text {. }
\end{aligned}
$$

The operator $\mathcal{L}^{u}$ is the infinitesimal generator associated to the stochastic process (II.2) [18, Section 7.3]. The symbols $\partial_{x}$ and $\partial_{x, x^{\prime}}$ denote first- and second-order partial derivatives with respect to $x$ and $x^{\prime}$, respectively.

It is known that a switching system whose subsystems are all stable, may exhibit some unstable behaviors under some switching signals [15], hence the overall system may not be stable in general. The same can happen for a switching stochastic system [4]. As a consequence, the $\delta$-GAS-M $\mathrm{M}_{q}$ property of switching stochastic systems can be established by using a common $\delta$-GAS-M ${ }_{q}$ Lyapunov function, or alternatively via multiple $\delta$-GAS-M M $_{q}$ Lyapunov functions that are mode dependent and under sojourn-time conditions.

Let the $\mathcal{K}_{\infty}$ functions $\underline{\alpha}, \bar{\alpha}$, and the constant $\kappa$ be defined as $\underline{\alpha}=\min \left\{\underline{\alpha}_{1}, \ldots, \underline{\alpha}_{m}\right\}, \bar{\alpha}=\max \left\{\bar{\alpha}_{1}, \ldots, \bar{\alpha}_{m}\right\}$, and $\kappa=\min \left\{\kappa_{1}, \ldots, \kappa_{m}\right\}$. Note that in the case of a common Lyapunov function, we have that $\underline{\alpha}=\underline{\alpha}_{1}=\cdots=\underline{\alpha}_{m}$ and $\bar{\alpha}=\bar{\alpha}_{1}=\cdots=\bar{\alpha}_{m}$. The following result provides a sufficient condition for a switching stochastic system $\Sigma$ to be $\delta$-GAS-M $\mathrm{M}_{q}$ based on the existence of a common $\delta$-GAS$\mathrm{M}_{q}$ Lyapunov function.

Theorem 3.3: Consider a switching stochastic system $\Sigma$. If there exists a common $\delta$-GAS-M M $_{q}$ Lyapunov function $V$ for all the subsystems $\left\{\Sigma_{1}, \ldots, \Sigma_{m}\right\}$, then $\Sigma$ is $\delta$-GAS-M ${ }_{q}$.

The existence of a common Lyapunov function in Theorem 3.3 may fail to hold in general. One can alternatively describe $\delta$-GAS-M $\mathrm{M}_{q}$ stability by resorting to multiple $\delta$-GAS$\mathrm{M}_{q}$ Lyapunov functions, under a class of switching signals that is fairly general and quite natural to consider.

Assumption 3.4: Consider the stochastic process $\widehat{\pi}: \Omega \times$ $\mathbb{R}_{0}^{+} \rightarrow \mathrm{P}$ on the probability space $(\Omega, \mathcal{F}, \mathbb{P})$ such that, for every fixed $\omega \in \Omega, \pi(\cdot)=\widehat{\pi}(\omega, \cdot): \mathbb{R}_{0}^{+} \rightarrow \mathrm{P}$ belongs to $\mathcal{P}$, and assume that $\widehat{\pi}$ is completely known at time $t=0$. We assume that there exists some $\lambda \in \mathbb{R}_{0}^{+}$such that for any $\widehat{\pi}$, the probability of sojourning (staying in a mode) within an infinitesimal time interval $h$ is lower-bounded by the following, for any $p \in \mathrm{P}$ :

$$
\mathbb{P}[\widehat{\pi}(t+h)=p \mid \widehat{\pi}(t)=p] \geq 1-\lambda h .
$$

Remark 3.5: If the switching process $\widehat{\pi}$ is characterised by a continuous-time Markov chain with a given generator matrix $Q=\left\{q_{i j}\right\} \in \mathbb{R}^{\mathrm{P} \times \mathrm{P}}$, one can obtain the lower bound on the probability in (III.2) as $\lambda=\max _{i \in \mathrm{P}} \sum_{i \neq j} q_{i j}$.

For a stochastic switching process $\widehat{\pi}$, we denote the number of switches (the discontinuity points of $\widehat{\pi}$ ) on the interval $] 0, t]$ by $N_{\widehat{\pi}}(t)$, which is measurable in $\mathcal{F}_{t}$. We assume $N_{\widehat{\pi}}(0)=0$. Due to Assumption 3.4 on $\widehat{\pi}$, the probability distribution of $N_{\widehat{\pi}}(t)$ satisfies [4]:

$$
\mathbb{P}\left[N_{\widehat{\pi}}(t)=k\right] \leq \frac{e^{-\lambda t}(\lambda t)^{k}}{k !} .
$$

From (III.3), one can readily verify that the counting process $\left\{N_{\widehat{\pi}}\right\}_{t \geq 0}$ takes with probability one finite values over finite time intervals. We assume that $\left\{W_{t}\right\}_{t \geq 0},\left\{N_{\widehat{\pi}}\right\}_{t \geq 0}$, and the initial condition of $\Sigma$, which is measurable in $\overline{\mathcal{F}}_{0}$, are mutually independent. The next result provides sufficient conditions for a switching stochastic system $\Sigma$ to be $\delta$-GAS$\mathrm{M}_{q}$ based on the existence of multiple $\delta$-GAS-M $\mathrm{M}_{q}$ Lyapunov functions and on Assumption 3.4.

Theorem 3.6: Consider a switching stochastic system $\Sigma$. Assume that Assumption 3.4 holds and that for any $p \in \mathrm{P}$, there exists a $\delta$-GAS-M $\mathrm{M}_{q}$ Lyapunov function $V_{p}$ for $\Sigma_{p}$, and in addition that there exits a constant $\mu \geq 1$ such that

(i) for any $x, x^{\prime} \in \mathbb{R}^{n}$, and any $p, p^{\prime} \in \mathrm{P}$,

$$
V_{p}\left(x, x^{\prime}\right) \leq \mu V_{p^{\prime}}\left(x, x^{\prime}\right)
$$

(ii) $(\mu-1) \lambda-\kappa<0$.

Then $\Sigma$ is $\delta$-GAS-M $\mathrm{M}_{q}$.

We refer the interested readers to the results in [25], providing special instances where these functions can be easily computed. For example, for linear stochastic subsystems (i.e. for subsystems with linear drift and diffusion terms), one can search for appropriate $\delta$-GAS-M ${ }_{q}$ Lyapunov functions by easily solving linear matrix inequalities (LMI).

In order to show the main result of the paper, we need the following technical lemma, borrowed from [25], which provides an upper bound on the distance (in the $q$ th moment metric) between the solution processes of subsystems $\Sigma_{p}$ and the corresponding non-probabilistic subsystems obtained by disregarding the diffusion term $\left(g_{p}\right)$. From now on, we use the notation $\zeta_{x v}^{p}$ to denote the solution of the ordinary differential equation (ODE) $\dot{\zeta}_{x v}^{p}=f_{p}\left(\zeta_{x v}^{p}, v\right)$ starting from the initial condition $x$ and under the input curve $v$.

Lemma 3.7: Consider a stochastic subsystem $\Sigma_{p}$ such that $f\left(0_{n}, 0_{m}\right)=0_{n}$ and $g_{p}\left(0_{n}\right)=0_{n \times \widehat{q}}$. Suppose that $q \geq 2$ and that there exists a $\delta$-GAS-M $\mathrm{M}_{q}$ Lyapunov function $V_{p}$ for $\Sigma_{p}$ such that its Hessian is a positive semidefinite matrix in $\mathbb{R}^{2 n \times 2 n}$ and $\partial_{x, x} V_{p}\left(x, x^{\prime}\right) \leq P_{p}$, for any $x, x^{\prime} \in \mathbb{R}^{n}$, and some positive semidefinite matrix $P_{p} \in \mathbb{R}^{n \times n}$. Then for any $x$ in a compact set $\mathrm{D} \subset \mathbb{R}^{n}$ and any $v \in \mathcal{U}$, we have

$$
\mathbb{E}\left[\left\|\xi_{x v}^{p}(t)-\zeta_{x v}^{p}(t)\right\|^{q}\right] \leq h_{p}\left(g_{p}, t\right),
$$

where the nonnegative valued function $h_{p}$ tends to zero as $t \rightarrow 0, t \rightarrow+\infty$, or as $Z \rightarrow 0$, where $Z$ is the Lipschitz constant introduced in Definition 2.1. 
In particular, one can compute explicitly the function $h_{p}$ using equation (9.4) in [25]. For later use, we introduce function $h(G, t)=\max \left\{h_{1}\left(g_{1}, t\right), \ldots, h_{m}\left(g_{m}, t\right)\right\}$ for all $t \in \mathbb{R}_{0}^{+}$.

\section{SYSTEMS AND \\ APPROXIMATE EQUIVALENCE RELATIONS}

We employ the notion of system, introduced in [22], to provide (in Sec. V) an alternative description of switching stochastic systems that will be directly related to their finite abstractions.

Definition 4.1: A system $S$ is a tuple $S=\left(X, X_{0}, U, \longrightarrow\right.$ $, Y, H)$, where

- $X$ is a set of states (possibly infinite);

- $X_{0} \subseteq X$ is a set of initial states (possibly infinite);

- $U=A \times B$ is a set of inputs, where

- $A$ is the set of control inputs (possibly infinite);

- $B$ is the set of adversarial inputs (possibly infinite);

- $\longrightarrow \subseteq X \times U \times X$ is a transition relation;

- $Y$ is a set of outputs;

- $H: X \rightarrow Y$ is an output map.

We write $x \stackrel{a, b}{\longrightarrow} x^{\prime}$ if $\left(x,(a, b), x^{\prime}\right) \in \longrightarrow$. If $x \stackrel{a, b}{\longrightarrow} x^{\prime}$, we call state $x^{\prime}$ a successor of state $x$. For technical reasons, we assume that for each $x \in X$, there is some successor of $x$, for some $(a, b) \in U-$ let us remark that this is always the case for the systems considered later in this paper.

A system $S$ is said to be

- metric, if the output set $Y$ is equipped with a metric $\mathbf{d}: Y \times Y \rightarrow \mathbb{R}_{0}^{+}$

- countable, if $X$ and $U$ are countable sets;

- finite (or symbolic), if $X$ and $U$ are finite sets.

For a system $S=\left(X, X_{0}, U, \longrightarrow, Y, H\right)$ and given any initial state $x_{0} \in X_{0}$, a finite state run generated from $x_{0}$ is a finite sequence of transitions:

$$
x_{0} \stackrel{a_{0}, b_{0}}{\longrightarrow} x_{1} \stackrel{a_{1}, b_{1}}{\longrightarrow} \cdots x_{n-1} \stackrel{a_{n-1}, b_{n-1}}{\longrightarrow} x_{n},
$$

such that $x_{i} \stackrel{a_{i}, b_{i}}{\longrightarrow} x_{i+1}$ for all $0 \leq i<n$. A finite state run can be trivially extended to an infinite state run. A finite output run is a sequence $\left\{y_{0}, y_{1}, \ldots, y_{n}\right\}$ such that there exists a finite state run of the form (IV.1) with $y_{i}=H\left(x_{i}\right)$, for $i=0, \ldots, n$. A finite output run can also be directly extended to an infinite output run.

We recall the notion of alternating approximate (bi)simulation relation, as discussed in [19], which captures the different role of control and adversarial inputs in the systems.

Definition 4.2: Let $S_{1}=\left(X_{1}, X_{10}, A_{1} \times\right.$ $\left.B_{1}, \longrightarrow, Y_{1}, H_{1}\right)$ and $S_{2}=\left(X_{2}, X_{20}, A_{2} \times\right.$ $\left.B_{2}, \underset{2}{\longrightarrow}, Y_{2}, H_{2}\right)$ be metric systems with the same output sets $Y_{1}=Y_{2}$ and metric $\mathbf{d}$. For $\varepsilon \in \mathbb{R}_{0}^{+}$, a relation $R \subseteq X_{1} \times X_{2}$ is said to be an alternating $\varepsilon$-approximate simulation relation from $S_{1}$ to $S_{2}$ if the following three conditions are satisfied:

(i) for every $x_{10} \in X_{10}$, there exists $x_{20} \in X_{20}$ with $\left(x_{10}, x_{20}\right) \in R$ (ii) for every $\left(x_{1}, x_{2}\right) \in R, \mathbf{d}\left(H_{1}\left(x_{1}\right), H_{2}\left(x_{2}\right)\right) \leq \varepsilon$;

(iii) for every $\left(x_{1}, x_{2}\right) \in R, \forall a_{1} \in A_{1} \exists a_{2} \in A_{2} \forall b_{2} \in B_{2}$ $\exists b_{1} \in B_{1}$ such that $x_{1} \underset{1}{\stackrel{a_{1}, b_{1}}{\longrightarrow}} x_{1}^{\prime}$ and $x_{2} \underset{2}{\stackrel{a_{2}, b_{2}}{\longrightarrow}} x_{2}^{\prime}$ with $\left(x_{1}^{\prime}, x_{2}^{\prime}\right) \in R$.

A relation $R \subseteq X_{1} \times X_{2}$ is said to be an alternating $\varepsilon$ approximate bisimulation relation between $S_{1}$ and $S_{2}$ if $R$ is an alternating $\varepsilon$-approximate simulation relation from $S_{1}$ to $S_{2}$ and $R^{-1}$ is an alternating $\varepsilon$-approximate simulation relation from $S_{2}$ to $S_{1}$.

System $S_{1}$ is alternatingly $\varepsilon$-approximately simulated by $S_{2}$, or $S_{2}$ alternatingly $\varepsilon$-approximately simulates $S_{1}$, denoted by $S_{1} \preceq_{\mathcal{A S S}}^{\varepsilon} S_{2}$, if there exists an alternating $\varepsilon$ approximate simulation relation from $S_{1}$ to $S_{2}$. System $S_{1}$ is alternatingly $\varepsilon$-approximately bisimilar to $S_{2}$, denoted by $S_{1} \cong{ }_{\mathcal{A S}} S_{2}$, if there exists an alternating $\varepsilon$-approximate bisimulation relation between $S_{1}$ and $S_{2}$.

\section{Finite ABStRaCtions FOR SWITCHING STOCHASTIC SYSTEMS}

This section contains the main contribution of this work. We show that for any $\delta$-GAS-M $q$ switching stochastic system $\Sigma$, initialized within a bounded set, and for any precision level $\varepsilon \in \mathbb{R}^{+}$, there exists a finite system that is alternatingly $\varepsilon$-approximately bisimilar to $\Sigma$. In order to do so, we use systems as an abstract representation of switching stochastic systems. More precisely, given a switching stochastic system $\Sigma$ and a sampling time $\tau \in \mathbb{R}^{+}$, we define the associated system $S_{\tau}(\Sigma)=\left(X_{\tau}, X_{\tau 0}, U_{\tau}, \longrightarrow \tau, Y_{\tau}, H_{\tau}\right)$, where

- $X_{\tau}$ is the set of all $\mathbb{R}^{n}$-valued random variables defined on the probability space $(\Omega, \mathcal{F}, \mathbb{P})$;

- $X_{\tau 0}$ is the set of all $\mathbb{R}^{n}$-valued random variables that are measurable over the trivial sigma-algebra $\mathcal{F}_{0}$, i.e. the system starts from a non-probabilistic initial condition;

- $U_{\tau}=A_{\tau} \times B_{\tau}$, where

- $A_{\tau}=\{v \in \mathcal{U} \mid$ the domain of $v$ is $[0, \tau[\}$;

- $B_{\tau}=\{\pi \in \mathcal{P} \mid$ the domain of $\pi$ is $[0, \tau[\}$;

- $x_{\tau} \underset{\tau}{\stackrel{v_{\tau}, \pi_{\tau}}{\longrightarrow}} x_{\tau}^{\prime}$ if $x_{\tau}$ and $x_{\tau}^{\prime}$ are measurable, respectively, in $\mathcal{F}_{k \tau}$ and $\mathcal{F}_{(k+1) \tau}$ for some $k \in \mathbb{N}_{0}$, and there exists a solution process $\xi: \Omega \times \mathbb{R}_{0}^{+} \rightarrow \mathbb{R}^{n}$ of $\Sigma$ satisfying $\xi(k \tau)=x_{\tau}$ and $\xi_{x_{\tau} v_{\tau}}^{\pi_{\tau}}(\tau)=x_{\tau}^{\prime}$ P-a.s.;

- $Y_{\tau}=X_{\tau}$;

- $H=1_{X_{\tau}}$.

Note that a finite state run $x_{0} \stackrel{v_{0}, \pi_{0}}{\longrightarrow} x_{1} \stackrel{v_{1}, \pi_{1}}{\tau} \cdots \frac{v_{N-1}, \pi_{N-1}}{\tau} x_{N}$ of $S_{\tau}(\Sigma)$, where $v_{i-1} \in A_{\tau}, \pi_{i-1} \in B_{\tau}$, and $x_{i}=\xi_{x_{i-1} v_{i-1}}^{\pi_{i-1}}(\tau)$ P-a.s. for $i=1, \ldots, N$, captures the trajectory of the switching stochastic system $\Sigma$ at times $t=0, \tau, \ldots, N \tau$. This trajectory starts from the non-probabilistic initial condition $x_{0}$ and results from the control input $v$ and the adversarial input (or switching signal) $\pi$ obtained by the concatenation of the control and adversarial inputs $v_{i-1}$ and $\pi_{i-1}$, respectively, (that is, $v((i-1) \tau+s)=v_{i-1}(s)$ and $\pi((i-1) \tau+s)=\pi_{i-1}(s)$ for any $s \in[0, \tau[)$, for $i=1, \ldots, N$.

Given a switching stochastic system $\Sigma=$ $\left(\mathbb{R}^{n}, \mathrm{U}, \mathcal{U}, \mathrm{P}, \mathcal{P}, F, G\right)$, we define for subsequent analysis 
the corresponding switching non-probabilistic system $\bar{\Sigma}=\left(\mathbb{R}^{n}, \mathrm{U}, \mathcal{U}, \mathrm{P}, \mathcal{P}, F\right)$, defined by the ODE $\dot{\zeta}=f_{\pi}(\zeta, v)$, for any $v \in \mathcal{U}$ and any $\pi \in \mathcal{P}$. Note that due to the assumptions on $f_{p}$, for any $p \in \mathrm{P}$, each subsystem $\bar{\Sigma}_{p}$ of $\bar{\Sigma}$ is forward complete [3], i.e. every trajectory is defined on the interval $[0, \infty[$. Moreover, due to the assumptions on switching signals $\pi \in \mathcal{P}$, one can conclude that the overall switching non-probabilistic system $\bar{\Sigma}$ is forward complete ${ }^{1}$ [17]. We also write $\zeta_{x v}^{\pi}(t)$ to denote the point reached at time $t \in \mathbb{R}_{0}^{+}$under the control input $v \in \mathcal{U}$ and the switching signal $\pi$ from the initial condition $\zeta_{x v}^{\pi}(0)=x$.

In order to construct a symbolic model for any $\delta$-GAS-M switching stochastic system $\Sigma$, we will extract a countable set of inputs $U_{\mathrm{g}}$ from $U_{\tau}$ in such a way that the resulting symbolic model is countable and indeed finite if we are interested in the dynamics of $\Sigma$ initialized within a bounded set. Note that the approximation of the set of inputs $U_{\tau}$ of $S_{\tau}(\Sigma)$ requires the notion of reachable set, as defined next. Given a switching non-probabilistic system $\bar{\Sigma}$, any $\tau \in \mathbb{R}^{+}$, and $x \in \mathbb{R}^{n}$, the reachable set of $\bar{\Sigma}$ with initial condition $x \in \mathbb{R}^{n}$ after $\tau$ seconds is the set $\mathcal{R}(\tau, x)$ of endpoints $\zeta_{x v}^{\pi}(\tau)$ for any $v \in A_{\tau}$ and $\pi \in B_{\tau}$ or, equivalently,

$$
\mathcal{R}(\tau, x):=\left\{y \in \mathbb{R}^{n} \mid y=\zeta_{x v}^{\pi}(\tau), v \in A_{\tau}, \pi \in B_{\tau}\right\} .
$$

Moreover, the reachable set of $\bar{\Sigma}$ with initial condition $x \in$ $\mathbb{R}^{n}$ and control input $v \in A_{\tau}$ after $\tau$ seconds is the set $\mathcal{R}(\tau, x, v)$ of endpoints $\zeta_{x v}^{\pi}(\tau)$ for any $\pi \in B_{\tau}$, i.e.,

$$
\mathcal{R}(\tau, x, v):=\left\{y \in \mathbb{R}^{n} \mid y=\zeta_{x v}^{\pi}(\tau), \pi \in B_{\tau}\right\} .
$$

The reachable sets in (V.1) and (V.2) are well defined because $\bar{\Sigma}$ is forward complete. Given any desired precision $\mu \in \mathbb{R}^{+}$and $\eta \in \mathbb{R}^{+}$and following the same approach in [19], we approximate $U_{\tau}$ in the definition of $S_{\tau}(\Sigma)$ by means of the set $U_{\mathrm{q}}:=A_{\mathrm{q}} \times B_{\mathrm{q}}$, where

$A_{\mathrm{q}}:=\cup_{x_{\mathrm{q}} \in X_{\mathrm{q}}} A^{\mu}\left(x_{\mathrm{q}}\right), B_{\mathrm{q}}:=\cup_{x_{\mathrm{q}} \in X_{\mathrm{q}}} \cup_{v \in A^{\mu}\left(x_{\mathrm{q}}\right)} B^{\mu}\left(x_{\mathrm{q}}, v\right)$,

and where $X_{\mathrm{q}}=\left[\mathbb{R}^{n}\right]_{\eta}, A^{\mu}\left(x_{\mathrm{q}}\right)$ captures the set of control inputs that can be applied at state $x_{\mathrm{q}} \in X_{\mathrm{q}}$, while $B^{\mu}\left(x_{\mathrm{q}}, v\right)$ captures the set of switching signals that can be applied at the state $x_{\mathrm{q}} \in X_{\mathrm{q}}$ when the chosen control input is $v \in A^{\mu}\left(x_{\mathrm{q}}\right)$. Given any $\tau \in \mathbb{R}^{+}$, define the following sets:

$$
\begin{aligned}
& \mathrm{A}_{\mu}\left(\tau, x_{\mathrm{q}}\right):= \\
& \quad\left\{P \in 2^{\left[\mathbb{R}^{n}\right]_{\mu}} \mid \exists v \in A_{\tau} \text { s.t. } \mathbf{d}_{h}\left(P, \mathcal{R}\left(\tau, x_{\mathrm{q}}, v\right)\right) \leq \mu\right\}, \\
& \mathrm{B}_{\mu}\left(\tau, x_{\mathrm{q}}, v\right):= \\
& \quad\left\{x_{\mathrm{q}}^{\prime} \in\left[\mathbb{R}^{n}\right]_{\mu} \mid \exists \pi \in B_{\tau} \text { s.t. }\left\|x_{\mathrm{q}}^{\prime}-\zeta_{x_{\mathrm{q} v}}^{\pi}(\tau)\right\| \leq \mu\right\},
\end{aligned}
$$

where $\mathbf{d}_{h}$ is the Hausdorff pseudometric induced by the infinity norm on $\mathbb{R}^{n}$. Note that for any $P \in \mathrm{A}_{\mu}\left(\tau, x_{\mathrm{q}}\right)$ and any $x_{\mathrm{q}}^{\prime} \in \mathrm{B}_{\mu}\left(\tau, x_{\mathrm{q}}, v\right)$, there may exist a (possibly uncountable) set of control inputs $v \in A_{\tau}$ and a (possibly uncountable) set of switching signals $\pi \in B_{\tau}$ such that $\mathbf{d}_{h}\left(P, \mathcal{R}\left(\tau, x_{\mathrm{q}}, v\right)\right) \leq \mu$ and $\left\|x_{\mathrm{q}}^{\prime}-\zeta_{x_{\mathrm{q}} v}^{\pi}(\tau)\right\| \leq \mu$, respectively. One can construct countable (possibly finite) sets of control inputs and switching signals by just collecting some

\footnotetext{
${ }^{1}$ Note that if one had allowed for Zeno behavior in $\bar{\Sigma}$, it might have caused finite escape time even if all the subsystems were forward complete.
}

representative ones, as explained in the following. Let us define the functions

$$
\psi_{\mu}^{\tau, x_{\mathrm{q}}}: \mathrm{A}_{\mu}\left(\tau, x_{\mathrm{q}}\right) \rightarrow A_{\tau}, \varphi_{\mu}^{\tau, x_{\mathrm{q}}, v}: \mathrm{B}_{\mu}\left(\tau, x_{\mathrm{q}}, v\right) \rightarrow B_{\tau},
$$

where

- $\psi_{\mu}^{\tau, x_{\mathrm{q}}}$ associates to any $P \in \mathrm{A}_{\mu}\left(\tau, x_{\mathrm{q}}\right)$ one control input $v \in \psi_{\mu}^{\tau, x_{\mathrm{q}}}(P) \in A_{\tau}$ so that $\mathbf{d}_{h}\left(P, \mathcal{R}\left(\tau, x_{\mathrm{q}}, v\right)\right) \leq \mu$

- $\varphi_{\mu}^{\tau, x_{\mathrm{q}}, v}$ associates to any $x_{\mathrm{q}}^{\prime} \in \mathrm{B}_{\mu}\left(\tau, x_{\mathrm{q}}, v\right)$ one switching signal $\pi=\varphi_{\mu}^{\tau, x_{\mathrm{q}}, v}\left(x_{\mathbf{q}}^{\prime}\right) \in B_{\tau}$ so that $\left\|x_{\mathrm{q}}^{\prime}-\zeta_{x_{\mathrm{q}} v}^{\pi}(\tau)\right\| \leq \mu$.

Note that functions $\psi_{\mu}^{\tau, x_{\mathrm{q}}}$ and $\varphi_{\mu}^{\tau, x_{\mathrm{q}}, v}$ are not unique.

Now one can define the sets $A^{\mu}\left(x_{\mathbf{q}}\right)$ and $B^{\mu}\left(x_{\mathbf{q}}, v\right)$ appearing in (V.3) as follows:

$$
\begin{aligned}
A^{\mu}\left(x_{\mathrm{q}}\right) & :=\psi_{\mu}^{\tau, x_{\mathrm{q}}}\left(\mathrm{A}_{\mu}\left(\tau, x_{\mathrm{q}}\right)\right), \\
B^{\mu}\left(x_{\mathrm{q}}, v\right) & :=\varphi_{\mu}^{\tau, x_{\mathrm{q}}, v}\left(\mathrm{~B}_{\mu}\left(\tau, x_{\mathrm{q}}, v\right)\right) .
\end{aligned}
$$

We remark again that since $\bar{\Sigma}$ is forward complete, sets $\mathrm{A}_{\mu}\left(\tau, x_{\mathrm{q}}\right)$ and $\mathrm{B}_{\mu}\left(\tau, x_{\mathrm{q}}, v\right)$ in (V.4) and (V.5) are nonempty and therefore $A^{\mu}\left(x_{\mathrm{q}}\right)$ and $B^{\mu}\left(x_{\mathrm{q}}, v\right)$ in (V.7) and (V.8) are nonempty, as well.

We now have all the ingredients to introduce a symbolic model for $S_{\tau}(\Sigma)$.

Consider a switching stochastic system $\Sigma$, and a triple $\mathrm{q}=$ $(\tau, \eta, \mu)$ of quantization parameters, where $\tau$ is the sampling time, $\eta$ is the state-space quantization, and $\mu$ is a design parameter. Given $\Sigma$ and q, consider the following system:

$$
S_{\mathrm{q}}(\Sigma)=\left(X_{\mathrm{q}}, X_{\mathrm{q} 0}, U_{\mathrm{q}}, \underset{\mathrm{q}}{\longrightarrow}, Y_{\mathrm{q}}, H_{\mathrm{q}}\right),
$$

where $X_{\mathrm{q}}=\left[\mathbb{R}^{n}\right]_{\eta}, X_{\mathrm{q} 0}=\left[\mathbb{R}^{n}\right]_{\eta}$, and

- $U_{\mathrm{q}}=A_{\mathrm{q}} \times B_{\mathrm{q}}$, where

$$
A_{\mathrm{q}}=\cup_{x_{\mathrm{q}} \in X_{\mathrm{q}}} A^{\mu}\left(x_{\mathrm{q}}\right), B_{\mathrm{q}}=\cup_{x_{\mathrm{q}} \in X_{\mathrm{q}}} \cup_{v \in A^{\mu}\left(x_{\mathrm{q}}\right)} B^{\mu}\left(x_{\mathrm{q}}, v\right) \text {, }
$$

and the sets $A^{\mu}\left(x_{\mathbf{q}}\right)$ and $B^{\mu}\left(x_{\mathbf{q}}, v\right)$ are defined in (V.7) and (V.8), respectively;

- $x_{\mathrm{q}} \underset{\mathrm{q}}{\stackrel{v_{\mathrm{q}}, \pi_{\mathrm{q}}}{\longrightarrow}} x_{\mathrm{q}}^{\prime}$ if $v_{\mathrm{q}} \in A^{\mu}\left(x_{\mathrm{q}}\right), \pi_{\mathrm{q}} \in B^{\mu}\left(x_{\mathrm{q}}, v_{\mathrm{q}}\right)$, and there exists $x_{\mathrm{q}}^{\prime} \in X_{\mathrm{q}}$ such that $\left\|\zeta_{x_{\mathrm{q}} v_{\mathrm{q}}}^{\pi_{\mathrm{q}}}(\tau)-x_{\mathrm{q}}^{\prime}\right\| \leq \eta$;

- $Y_{\mathrm{q}}$ is the set of all $\mathbb{R}^{n}$-valued random variables defined on the probability space $(\Omega, \mathcal{F}, \mathbb{P})$;

- $H_{\mathrm{q}}=\imath: X_{\mathrm{q}} \hookrightarrow Y_{\mathrm{q}}$.

Note that in the definition of $H_{\mathrm{q}}$, the inclusion map $\imath$ is meant, with a slight abuse of notation, as a mapping from a grid point to a random variable with a Dirac probability distribution centered at that grid point.

The transition relation of $S_{\mathrm{q}}(\Sigma)$ is well defined in the sense that for every $x_{\mathrm{q}} \in\left[\mathbb{R}^{n}\right]_{\eta}$, every $v_{\mathrm{q}} \in A^{\mu}\left(x_{\mathrm{q}}\right)$, and every $\pi_{\mathrm{q}} \in B^{\mu}\left(x_{\mathrm{q}}, v_{\mathrm{q}}\right)$, there always exists $x_{\mathrm{q}}^{\prime} \in\left[\mathbb{R}^{n}\right]_{\eta}$ such that $x_{\mathrm{q}} \underset{\mathrm{q}}{\stackrel{v_{\mathrm{q}}, \pi_{\mathrm{q}}}{\longrightarrow}} x_{\mathrm{q}}^{\prime}$. This can be easily shown, since by definition of $\left[\mathbb{R}^{n}\right]_{\eta}$, for any $\widehat{x} \in \mathbb{R}^{n}$ there always exists a state $\widehat{x}^{\prime} \in\left[\mathbb{R}^{n}\right]_{\eta}$ such that $\left\|\widehat{x}-\widehat{x}^{\prime}\right\| \leq \eta$. Hence, for $\zeta_{x_{\mathrm{q}} v_{\mathrm{q}}}^{\pi_{\mathrm{q}}}(\tau)$ there always exists a state $x_{\mathrm{q}}^{\prime} \in\left[\mathbb{R}^{n}\right]_{\eta}$ satisfying $\left\|\zeta_{x_{\mathrm{q}} v_{\mathrm{q}}}^{\pi_{\mathrm{q}}}(\tau)-x_{\mathrm{q}}^{\prime}\right\| \leq \eta$.

Before showing the main result of the paper, we need the following technical result.

Proposition 5.1: Consider a switching non-probabilistic system $\bar{\Sigma}=\left(\mathbb{R}^{n}, \mathrm{U}, \mathcal{U}, \mathrm{P}, \mathcal{P}, F\right)$. For any $x \in \mathbb{R}^{n}$ the reachable set $\mathcal{R}(\tau, x)$, defined in (V.1), is bounded. 
Note that $X_{\mathrm{q}}$ is a countable set. Since $\mathcal{R}(\tau, x)$, defined in (V.1), is bounded (cf. Proposition 5.1) and using Proposition 4.4 in [19], one can readily verify that $U_{\mathrm{q}}$ is also a countable set. Therefore, $S_{\mathrm{q}}(\Sigma)$ is countable. Moreover, if we are interested in the dynamics of $\Sigma$ in a bounded set, which is often the case in many practical situations, $S_{\mathrm{q}}(\Sigma)$ is finite.

We can now present the main result of the paper, which shows that any $\delta$-GAS-M ${ }_{q}$ switching stochastic system $\Sigma$ admits an alternatingly approximately bisimilar symbolic model.

Theorem 5.2: Consider a $\delta$-GAS-M $\mathrm{M}_{q}$ switching stochastic system $\Sigma$, satisfying the result in Lemma 3.7. For any $\varepsilon \in$ $\mathbb{R}^{+}$, and any triple $\mathbf{q}=(\tau, \eta, \mu)$ of quantization parameters satisfying

$$
\left(\beta\left(\varepsilon^{q}, \tau\right)\right)^{\frac{1}{q}}+(h(G, \tau))^{\frac{1}{q}}+2 \mu+\eta<\varepsilon,
$$

it holds that $S_{\mathrm{q}}(\Sigma) \cong{ }_{\mathcal{A S}}^{\varepsilon} S_{\tau}(\Sigma)$.

It can be readily seen that when we are interested in the dynamics of $\Sigma$ initialized in a compact $\mathrm{D} \subset \mathbb{R}^{n}$ of the form of a finite union of boxes, and for a given precision $\varepsilon$, there always exists a sufficiently large value of $\tau$ and small values of $\eta$ and $\mu$, such that $\eta \leq \operatorname{span}(\mathrm{D})$ and the condition in (V.10) are satisfied.

Remark 5.3: Note that in order to show the result in Theorem 5.2, one does not require any probabilistic structure on switching signals $\pi \in \mathcal{P}$, as long as the switching stochastic system $\Sigma$ admits a common $\delta$-GAS-M M $_{q}$ Lyapunov function or satisfies property (III.1) with some $\mathcal{K} \mathcal{L}$ function $\beta$. However, Assumption 3.4 allows us to compute the $\mathcal{K} \mathcal{L}$ function $\beta$, satisfying (III.1), by resorting to multiple $\delta$-GAS$\mathrm{M}_{q}$ Lyapunov functions.

\section{Conclusions}

In this paper we have shown the existence of symbolic models that are alternatingly approximately bisimilar to $\delta$ GAS-M $_{q}$ switching stochastic systems, for any $q \geq 1$. Moreover, we have provided a description of the $\delta$-GAS-M $\mathrm{M}_{q}$ property using a common $\delta$-GAS-M $\mathrm{M}_{q}$ Lyapunov function or,

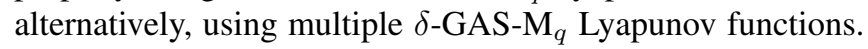
In future work we plan to focus on constructive approaches to obtain the symbolic models, the existence of which has been shown in this work.

\section{REFERENCES}

[1] A. Abate. A contractivity approach for probabilistic bisimulations of diffusion processes. In Proceedings of 48th IEEE Conference on Decision and Control, pages 2230-2235, December 2009.

[2] D. Angeli. A Lyapunov approach to incremental stability properties. IEEE Transactions on Automatic Control, 47(3):410-21, March 2002.

[3] D. Angeli and E. D. Sontag. Forward completeness, unboundedness observability, and their Lyapunov characterizations. Systems and Control Letters, 38:209-217, 1999.

[4] D. Chatterjee. Studies on stability and stabilization of randomly switched systems. $\mathrm{PhD}$ thesis, University of Illinois at UrbanaChampaign, 2007.

[5] A. Girard and G. J. Pappas. Approximation metrics for discrete and continuous systems. IEEE Transactions on Automatic Control, 25(5):782-798, May 2007.
[6] W. Glover and J. Lygeros. A stochastic hybrid model for air traffic control simulation. In R. Alur and G. J. Pappas, editors, Hybrid Systems: Computation and Control, volume 2993 of Lecture Notes in Computer Science, pages 372-386. Springer Berlin Heidelberg, March 2004.

[7] S. J. Hatjispyros and A. Yannacopoulos. A random dynamical system model of a stylized equity market. Physica A: Statistical and Theoretical Physics, 347:583-612, 2005.

[8] J. P. Hespanha. Stochastic hybrid systems: Application to communication networks. In R. Alur and G. J. Pappas, editors, Hybrid Systems: Computation and Control, volume 2993 of Lecture Notes in Computer Science, pages 387-401. Springer Berlin Heidelberg, March 2004.

[9] J. Hu, W. C. Wu, and S. Sastry. Modeling subtilin production in bacillus subtilis using stochastic hybrid systems. In R. Alur and G. J. Pappas, editors, Hybrid Systems: Computation and Control, volume 2993 of Lecture Notes in Computer Science, pages 417-431. Springer Berlin Heidelberg, March 2004.

[10] A. A. Julius and G. J. Pappas. Approximations of stochastic hybrid systems. IEEE Transaction on Automatic Control, 54(6):1193-1203, 2009.

[11] I. Karatzas and S. E. Shreve. Brownian Motion and Stochastic Calculus, volume 113 of Graduate Texts in Mathematics. SpringerVerlag, New York, 2nd edition, 1991.

[12] X. D. Koutsoukos. Optimal control of stochastic hybrid systems based on locally consistent markov decision processes. International Journal of Hybrid Systems, 4:301-318, 20042004.

[13] H. J. Kushner. Approximation and weak convergence methods for random processes with applications to stochastic systems theory. The MIT Press, July 2008.

[14] M. Lahijanian, S. B. Andersson, and C. Belta. A probabilistic approach for control of a stochastic system from LTL specifications. In Proceedings of 48th IEEE Conference on Decision and Control, pages 2236-2241, 2009.

[15] D. Liberzon. Switching in Systems and Control. Systems \& Control: Foundations \& Applications. Springer, 2003.

[16] O. Maler, A. Pnueli, and J. Sifakis. On the synthesis of discrete controllers for timed systems. In E. W. Mayr and C. Puech, editors, Symposium on Theoretical Aspects of Computer Science, volume 900 of LNCS, pages 229-242. Springer-Verlag, 1995.

[17] J. L. Mancilla-Aguilar, R. Garcia, E. D. Sontag, and Y. Wang. Representation of switched systems by perturbed control systems. In Proceedings of 43rd IEEE Conference on Decision and Control, pages 3259-3264, December 2004.

[18] B. K. Oksendal. Stochastic differential equations: An introduction with applications. Springer, 5th edition, November 2002.

[19] G. Pola and P. Tabuada. Symbolic models for nonlinear control systems: Alternating approximate bisimulations. SIAM Journal on Control and Optimization, 48(2):719-733, February 2009.

[20] J. Sproston. Discrete-time verification and control for probabilistic rectangular hybrid automata. In Proceedings of 8th International Conference on Quantitative Evaluation of Systems, pages 79-88, September 2011.

[21] A. M. Stankovic, G. C. Verghese, and D. J. Perrault. Analysis and synthesis of randomized modulation schemes for power converters. IEEE Transactions on Power Electronics, 10:680-693, 1995.

[22] P. Tabuada. Verification and Control of Hybrid Systems, A symbolic approach. Springer, 1st edition, June 2009.

[23] M. Zamani and A. Abate. Symbolic control of stochastic switched systems via finite abstractions. In K. Joshi, M. Siegle, M. Stoelinga, and P. R. D'Argenio, editors, Quantitative Evaluation of Systems, volume 8054 of Lecture Notes in Computer Science, pages 305-321. Springer Berlin Heidelberg, August 2013.

[24] M. Zamani, P. Mohajerin Esfahani, A. Abate, and J. Lygeros. Symbolic models for stochastic control systems without stability assumptions. In Proceedings of European Control Conference (ECC), pages 42574262, July 2013.

[25] M. Zamani, P. Mohajerin Esfahani, R. Majumdar, A. Abate, and J. Lygeros. Symbolic control of stochastic systems via approximately bisimilar finite abstractions. IEEE Transactions on Automatic Control, accepted, arXiv: 1302.3868, 2014.

[26] M. Zamani, I. Tkachev, and A. Abate. Bisimilar symbolic models for stochastic control systems without state-space discretization. In Proceedings of the 17th International Conference on Hybrid Systems: Computation and Control, April 2014, to appear. 